\title{
Tool Use and the Effect of Action on the Imagination
}

\author{
Daniel L. Schwartz and Douglas L. Holton \\ Vanderbilt University
}

\begin{abstract}
Three studies examined the claim that hand movements can facilitate imagery for object rotations but that this facilitation depends on people's model of the situation. In Experiment 1, physically turning a block without vision reduced mental rotation times compared with imagining the same rotation without bodily movement. In Experiment 2, pulling a string from a spool facilitated participants' mental rotation of an object sitting on the spool. In Experiment 3, depending on participants' model of the spool, the exact same pulling movement facilitated or interfered with the exact same imagery transformation. Results of Experiments 2 and 3 indicate that the geometric characteristics of an action do not specify the trajectory of an imagery transformation. Instead, they point to people's ability to model the tools that mediate between motor activity and its environmental consequences and to transfer tool knowledge to a new situation.
\end{abstract}

From hinges to hand drills, a distinctively human talent is the construction and use of multipart tools (Diamond, 1997). The purpose of this article is to explore a competence that may be responsible for this talent, namely, people's ability to imagine the environmental consequences of their actions. During tool use, objects often change position, for example, when a wrench turns a bolt. Imagery may help people anticipate the displacements that result from tool use. The literature on imagery points to this possibility, but it has primarily explored the functions of imagery for recognizing objects and spatial arrays displaced by an experimenter (e.g., Shelton \& McNamara, 1997; Shepard \& Cooper, 1986; Tarr \& Pinker, 1989). For imagery to enable tool use, it should also exhibit functions that specifically link the updating of an image to an individual's actions. Additionally, it should help people imagine how similar actions with different tools yield different physical changes. Our claim is that people's actions can facilitate their imagery for object displacements but that this facilitation depends on their mental model of the tool at hand.

It is a first principle of cognitive psychology that maps, plans, and other representations guide action in the environment. A useful capacity for tool use, however, would be if actions guided representations. Rieser, Garing, and Young (1994) and Simons and Wang (1998), for example, showed that walking without vision facilitates people's ability to imagine a spatial layout from another perspective. Similarly, Wexler, Kosslyn, and Berthoz (1998) and Wohlschlåger and Wolschlåger (1998) showed that physically turning an object without vision influences the ability to imagine the object at another orientation. Representations that respond to actions may help people coordinate their physical activity and mental expectations during tool use.

Daniel L. Schwartz and Douglas L. Holton, Department of Psychology and Human Development, Vanderbilt University.

We are deeply indebted to John Rieser for his pioneering work on action and representation and to Mary Hegarty for her continued interest.

Correspondence concerning this article should be addressed to Daniel $\mathrm{L}$. Schwartz, who is now at the School of Education, 485 Lasuen Mall, Stanford University, Stanford, California 94305-3096. Electronic mail may be sent to daniel.schwartz@stanford.edu.
One hypothesis for how action facilitates spatial updating is that there is a direct mapping between the geometry of a movement and the geometry of an imagined update. The assumption of this hypothesis is that the haptic information conveyed by an action specifies a spatially isomorphic effect on a representation (Gallistel, 1990). Each forward step of distance $d$ maps into an imagined forward distance of $d^{\prime}$. Turning one's hand $90^{\circ}$ specifies a $90^{\circ}$ update of the object in one's hand. The assumption of direct spatial mapping also occurs in motor theories of imagery in which a motor plan (as opposed to a perceived action) can drive imagery (see Sheerer, 1984 , for a review). The plan to turn one's hand clockwise, for example, may yield a clockwise update to the imagination.

The direct-mapping hypothesis is problematic in the context of tool use because it depends on a spatial isomorphism between action and representation. If actions always yielded spatially isomorphic imagery changes, tool use would be nearly impossible. When people turn the steering wheel of their car, they would anticipate a barrel roll instead of a right turn. The assumption that spatial similarities enable actions and motor plans to affect imagery may underestimate the flexibility of cognitive-level representations. Despite evidence that representations can comply to spatial constraints on action-for example, people's imagined hand movements honor the mechanics of their actual hand movements (Parsons, 1994)-tool imagery should not be a slave to action. With tools, people's actions can take one form, whereas the relevant spatial consequences can take a different form. Imagine, for example, that people pull a string from a spool, as shown in Figure 1. If they close their eyes, the linear action of pulling the string may help them imagine the rotation of the block.

For tool use, imagery should not only have the capacity to update with action; it should also be able to convert physical actions of one form into mental updates of another. We propose that mental models help people imagine how one movement causes a second movement within a physical situation and that imagery is the spatial face of a model (Hegarty, 1992; Schwartz \& Black, 1996). Mental models can incorporate spatial imagery plus the nonspatial information that helps people coordinate multiple transformations (Schwartz, 1999). For example, people can imag- 


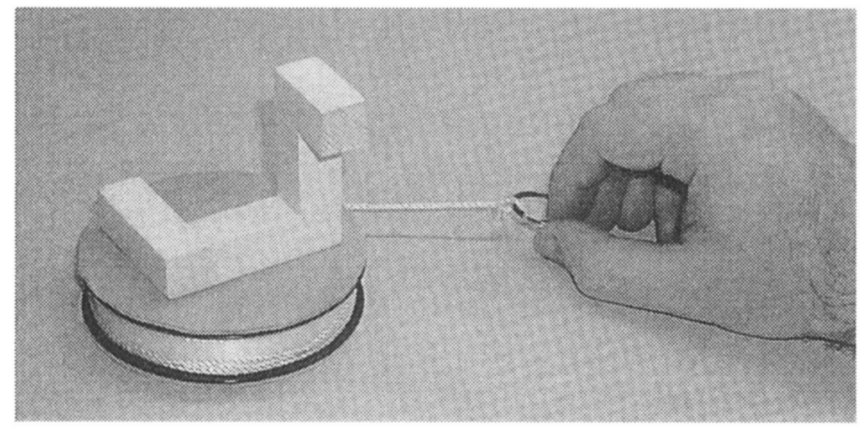

Figure 1. Can the linear motion of pulling the string help people imagine the rotation of the block atop the spool?

ine one gear driving another gear in the opposite direction by modeling the invisible friction between them (Schwartz \& Black, 1996). We propose that mental models also help people convert their bodily actions into imagery updates, updates that may or may not have the same spatial properties as the actions themselves.

Mental models may also help tool use because they enable people to reformulate the trajectory of an image depending on their knowledge of the tool at hand. This is important because the same action can be associated with different tools and outcomes. For example, if the string in Figure 1 were to unwind from the near side of the spool instead of the far side, the same pulling action would yield a counterclockwise motion instead of a clockwise one. People should be able to accommodate this change of outcome by constructing an appropriate model using their knowledge of the tool.

An ability to reformulate models so that the same action yields different imagery updates makes it difficult to maintain the hypothesis of a direct spatial mapping between action and imagery. Moreover, there is evidence that people's actions generate nonspatial information that guides their spatial updating, including resistance (Schwartz, 1999) and timing (Schwartz \& Berry, 2000). If the spatial properties of an action are insufficient to explain the effect of action on imagery, then it is useful to propose an alternative mechanism. Although we do not defend the proposal experimentally here, we argue elsewhere that people have timingresponsive representations (TRs; Schwartz, 1999; Schwartz \& Berry, 2000, Schwartz \& Black, 1999). As people operate in their environment, their actions generate timing signals indicative of change. TRs depend on these signals and update according to them. For each signal, a representation might update one step. Without the timing signals, it is more difficult to transform TRs. For example, people can draw some inferences through action that they cannot make through reflection, presumably because the action drives their representations (Krist, Fieberg, \& Wilkening, 1993). Schwartz and Black (1999) found that people are quite bad at determining whether a wide glass and a thin glass will pour at the same angle if filled to the same level of water. Yet, when given an opportunity to tilt empty glasses without vision and while imagining the behavior of the water, they correctly tilt a thin glass farther than a wide one. Presumably, the action of turning a glass generated the timing information that facilitated the imagined water movement.

Without the rhythm of action, people have to generate the timing of their mental transformations internally. For some tasks, this may be difficult to do in a coordinated manner. People may jump from one state to another in varying increments rather than in a smooth transformation. This reduces the resolution and continuity of their representational activity, and people have trouble completing certain types of tasks. This does not mean that people cannot simulate actions mentally (e.g., Decety \& Michel, 1989; de'Sperati \& Stucchi, 1997; Jeannerod, 1994) or simulate changes to distal objects, for example, through a mental rotation (Cooper \& Podgorny, 1976). These would be important abilities for designing new tools. Instead, it means that without the timing of action, continuous mental simulations are harder.

In sum, we propose a model in which action generates timing signals indicative of change. These signals drive imagery updates. The specific course of an imagery update depends on the mental model underlying the image. The subsequent experiments do not test all these proposals. Instead, they demonstrate three capacities that suggest model-mediated imagery could be responsible for multipart tool use. Experiment 1 shows that action can facilitate imagery. Experiment 2 shows that people can convert body movements of one trajectory into imagery updates of another. Experiment 3 shows that people's mental model of a tool determines their imagery update. In combination, these demonstrations show that actions can power representations to change but that representations themselves steer the direction of change. This conclusion helps counter the assumption that images and actions relate solely through a common geometry, and it encourages further investigations into the role of imagery in tool use. Moreover, by repealing the assumption of spatial isomorphism, the experiments create room for the TR alternative, an alternative that emphasizes the dynamics of action in real time.

\section{Experiment 1}

Each of the subsequent experiments asked participants to imagine the rotation of hand-sized objects. The first experiment showed that action increases the speed that people can imagine the rotation. It complements a number of investigations that indicate actions by the hands can interact with how people update their mental representations of distal objects (e.g., Kohler, 1964; Wexler \& Klam, in press; Wohlschlåger \& Wolschlåger, 1998; but see Wang \& Simons, 1999). The experiment used wooden versions of the computerized blocks used by Shepard and Metzler (1971) in their original mental rotation studies (see Figure 1). Participants, seated at a table across from the experimenter, completed four phases per trial.

During the encoding phase, participants examined a source block placed on the table. Once they felt they had the block sufficiently memorized, they grasped its midsection with the forefinger and thumb of their favored hand. The experimenter made sure the participants' fingers did not touch any part of the block that might provide information about the shape or position of its legs. Participants closed their eyes. The experimenter lowered a shelf that would conceal the block when participants reopened their eyes in the match phase. The experimenter placed a target block on the shelf, directly above the original source block. When the participants eventually opened their eyes, they had to determine whether this target block was the same as the source block or its 3-D reflection. 
In the precue phase, after participants had grasped the block and closed their eyes, they heard whether they should physically rotate the block or mentally rotate the block. After this instruction, they heard the direction of rotation (clockwise or counterclockwise $90^{\circ}$ along the plane of the table). In the action condition, they physically turned the block. In the no-action condition, they imagined the block without moving their hand.

The rotation phase began immediately after the precues. Once the participants completed the rotation, they opened their eyes. Latencies for the rotation phase began with the last word of the precue directions ("clockwise" or "counterclockwise") and ended when participants opened their eyes.

In the match phase, participants judged whether the target block on the shelf was the same as the source block. Latencies for the match phase began when participants opened their eyes and ended with the first word of their answer. The target block could appear in the expected position as described in the direction phase (rotated clockwise or counterclockwise $90^{\circ}$ ). It could also appear in the original (not rotated) position as seen during the encoding phase. If the participants actually rotated their block representation during the rotation phase, then their match times should be faster for the expected position than for the original position.

All told, each participant produced rotation and match latencies for eight correct match trials (Action/No-Action $\times$ Clockwise Counterclockwise $\times$ Expected/Original). If physically turning a block helps imagery, then rotation latencies should be shorter for the action trials than the no-action trials. If participants actually mentally rotate their block image before opening their eyes, latencies should be shorter for expected trials than original trials.

It is possible that the action condition could exhibit faster rotations than the no-action condition because participants might feel the shape of the rotated block in their hand. In this case, the action condition would facilitate the direct perception of a rotated block, not the imagination of a rotating block. Although we ensured that participants could not feel the legs of the block, it is possible that other sources of information could help them perceive the shape of the rotated block (e.g., inertia during movement; Turvey, Solomon, \& Burton, 1989). To ensure this was not the case, we added a substudy. In this study, participants did not see the source block at the start of each trial. Instead, they reached beneath the shelf, and their fingers were placed on the midsection of the block. With eyes closed, they physically rotated the block $90^{\circ}$. Afterward, they opened their eyes and saw a target block on the shelf in the rotated position. They had to decide if the target block was the same or a reflected version of the block in their hand. If participants cannot perceive the shape of the block during rotation, then they should perform at chance.

\section{Method}

Participants. Six women and 2 men volunteered for the main study. Additionally, 3 men and 3 women completed the perception substudy.

Design. The main study used a $2 \times 2$ within-subject design with three dependent measures. One measure was how long it took participants to complete a rotation. The second measure recorded how long it took participants to match the remembered source block against the target block. The third measure was accuracy. The Modality factor was whether participants completed an action or no-action rotation of a block. The Target factor was whether the target block was in the expected or original position. Participants completed each combination of the two factors twice, once going clockwise and once going counterclockwise. The order of trials was random. A "different" trial using a reflected block occurred randomly for one fourth of the trials to keep participants honest. The original and reflected blocks alternated as source blocks across trials. Blocks were set on the table in a variety of orientations. Only correct, same responses were included as data. When participants made a false response or stated they had lost their image, we replaced the bad trial with a second, identical trial at the end of the original trials.

In the perception substudy, there were eight same trials and eight different trials, equally consisting of clockwise and counterclockwise rotations of the source block. The target block was always in the expected position. The dependent measure was accuracy.

Procedure. Participants examined a block for as long as they needed to "get it in mind." They grasped the midpoint of the block and closed their eyes. Participants learned a sequence of precues that indicated the modality and direction of rotation. When simply imagining the rotation of the block, participants maintained their grasp of the block. Participants were asked to work quickly, but not so quickly that they would make a mistake. After practicing the rotation procedures, participants saw same and reflected blocks. They learned that after they opened their eyes they should make a same or different judgment. The participants practiced the complete sequence until they could follow the directives without hesitation. They then received the timed trials, measured with a lap stopwatch. After giving an answer, they examined the source and target blocks to see if they were right.

In the perception study, participants studied all the blocks for a few minutes before the trials. For a recorded trial, they closed their eyes and one of the blocks was placed between their fingers; we ensured that the participants did not touch the legs of the block. They physically rotated the block. They opened their eyes and judged whether the block in hand and the target block in the expected position were the same or reflected.

\section{Results}

Throughout the article, we adopt a significance level of .05 .

Perception study. We begin with the perception study to show that the subsequent action effects are not the result of "feeling" the answer to the problem. If no information comes from physically turning the blocks, participants would have to guess whether the block on the shelf matched the block in their hand. In contrast, if handling the block specifies its shape, then performance should exceed the chance level of $50 \%$ accuracy. The average performance was close to chance $(M=56 \%, S D=13 \%)$. Only 2 of the 6 participants were more than one answer removed from chance performance (11/16 and $12 / 16$ correct). It is possible these 2 participants were more sensitive to the available information. Regardless, handling the blocks does not appear to provide sufficient information for most participants to solve the problem. This result means that the effects of physically manipulating the blocks in the main study are unlikely to be the result of increased perceptual information that specified the answer to the problem.

Imagination study. Errors occurred on $9 \%$ of the trials. Three of the 8 participants made errors. Of their combined six mistakes, four were from the no-action condition. The errors distributed evenly over the expected and original trials. The generally accurate performance permits a focus on response latencies.

Figure 2 shows the mean rotation and match times broken down by modality and target condition. Participants completed the match faster when the target was in the expected position than when it was in the original position. This indicates that participants rotated their block representations before opening their eyes. Supporting 


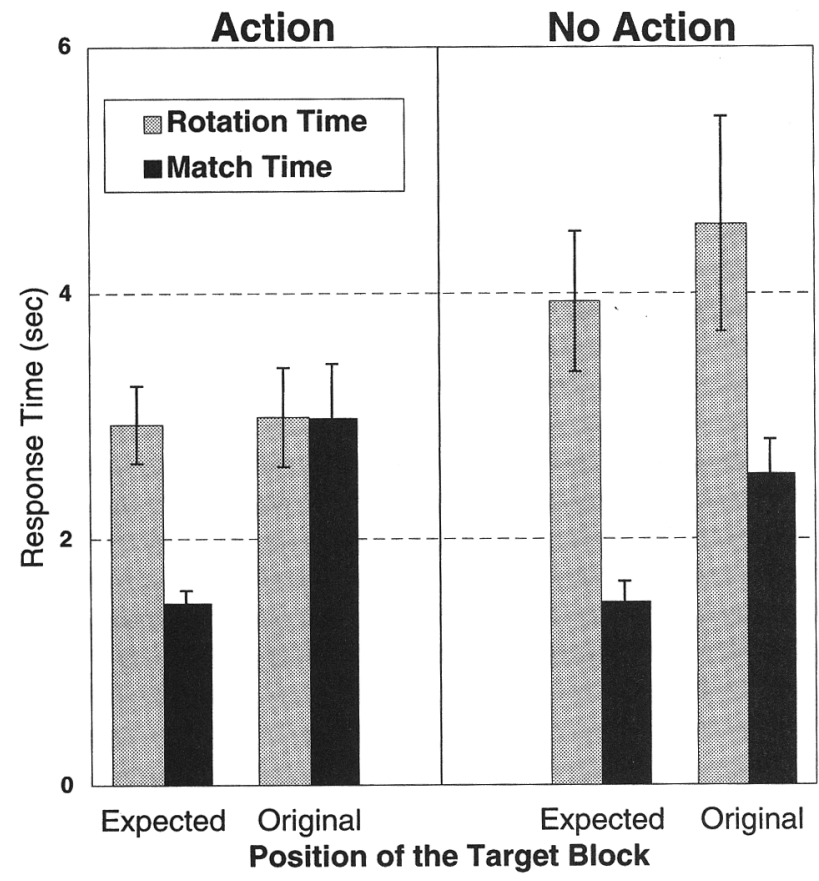

Figure 2. Latencies for the rotation and match phases of Experiment 1. $\sec =$ seconds.

the claim that action can drive representational updating, participants completed the rotations faster in the action condition than in the no-action condition. A within-subject analysis of variance compared rotation and match times for the crossed factors of Modality and Target. There was a significant effect of Modality on rotation time, $F(1,7)=8.3, M S E=1.6$, but not on match time, $F(1,7)=4.0, M S E=0.1$. There was a significant effect of Target on match time, $F(1,7)=24.5, M S E=0.5$, but it had no effect on rotation time, $F(1,7)=1.9, M S E=0.5$. There was no Modality $\times$ Target interaction on either rotation or match time, $F(2,6)=2.5$.

To explore the robustness of these results, we compared each action trial with its corresponding no-action trial (e.g., action/ clockwise/expected vs. no-action/clockwise/expected). Of the 32 comparisons across participants, 29 followed the predicted pattern in which the action rotation was faster than the yoked no-action rotation. We also compared the time to determine a match when the target block was in the expected or original position. Of the 32 pairs, 31 followed the predicted pattern in which matches were faster for the expected position than the original position.

The action rotations were also more consistent than the noaction rotations. All 8 participants exhibited more variability in their no-action rotation times than in their action rotations. The average standard deviation across a participant's no-action rotations was $0.97 \mathrm{~s}(S D=0.77)$. The average standard deviation for the action rotations was $0.58 \mathrm{~s}(S D=0.40)$. This difference is reliable in a one-tailed, paired $t$ test, $t(7)=2.3, S E=0.2$. There were no differences in the variability of the match times. Given that participants appeared to turn the blocks at a steady rate, this result suggests that the physical activity helped to "smooth" the imagery. This interpretation is tentative because one might expect a higher variability in the no-action condition simply because the response times were longer and provided more room for variance.

\section{Discussion}

When participants physically turned the source block, they increased the speed and consistency with which they could imagine its rotation. This shows that action can facilitate imagery. In both the action and no-action conditions, participants made faster judgments when the target block appeared at the rotated orientation than at the original orientation they encoded. This latter result serves as a manipulation check and demonstrates that participants did transform their representation of the source block before viewing the target. Our assumption is that participants completed an analog mental rotation of the source block to prepare for viewing the target. It is possible that they prepared themselves for the target block using a nonanalog approach, but it is difficult to explain why continuous action would help nonanalog processes.

The perception substudy helps to eliminate one interpretation of the results. This interpretation is that turning a block helped participants generate perceptual information that specified the block's configuration at its rotated position. The substudy showed that merely turning the block did not help participants feel whether the block in their hand was the same as the target. Action did not yield the perception of the block at its new orientation.

It is possible, however, that touching and turning the block provided enough perceptual information that it simplified the representational task. Feeling the block's movement may have improved the "cohesiveness" of its image (Wraga, Creen, \& Proffitt, 2000). Moreover, by turning the center of the block from horizontal to vertical, participants directly felt the block turning vertical, and therefore, they had no need to compute this transformation. Conceivably, this would lighten the processing burden and allow participants to speed their update of the representation overall. This interpretation is a variant of the direct-mapping hypothesis for the effect of action on representation. Action provides spatial information that is isomorphic to the changes participants need to make to their representations, and this isomorphism explains why actions support imagery. The next study attempted to show that this interpretation could not be fully correct. In Experiment 2, participants did not touch the source object, and their action took a different trajectory from the object. If action facilitated imagery for the object in this case, it is not due to added perceptual information that specifies properties of the object or its transformation.

\section{Experiment 2}

For this study, we invented an imagery task that we expected to be particularly sensitive to the benefits of action. Participants looked at three pegs of different colors set into a triangular pegboard (see Figure 3). After encoding the configuration and colors of the pegs, they closed their eyes. They received directions to rotate the pegs clockwise in their imagination until a specific peg was in the imagined line of sight to another specific peg (e.g., green obscures the view of red from where they are sitting). Once they indicated they had completed the rotation, they heard the names of two pegs (e.g., red and blue) and had to make a judgment of relative direction. With eyes still closed, they placed a ruler on the table parallel to the line made by the two pegs at their imagined position. The accuracy of the ruler's direction and the task latencies indicate the difficulty of the imagery. 
Rotate: Turn so green obscures red

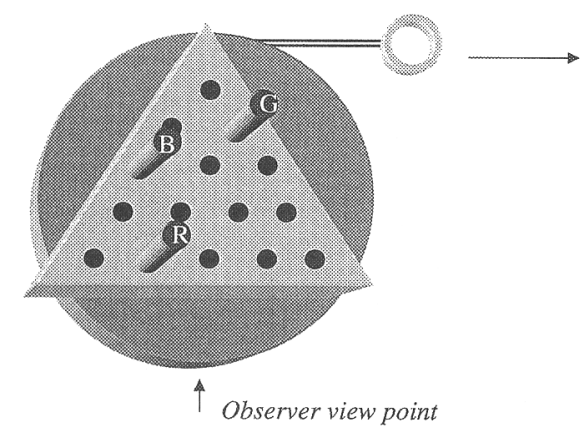

Judge: Show line made by red \& blue

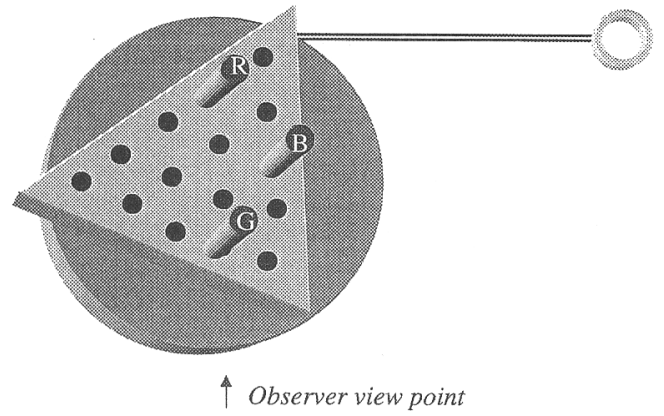

Figure 3. The pegboard task of Experiment 2.

The peg task is relatively novel, because participants neither see nor hear the necessary degree of rotation beforehand. For the peg task, participants have to attend to changes within their image to know when to stop (when the two pegs align). This differs from many imagery tasks in which the target indicates the angle of rotation or the angle is prespecified using an external frame of reference (e.g., turn the block $90^{\circ}$; see Schwartz \& Black, 1999). For these latter classes of problems, it is not necessary to complete the rotation in fine gradations (e.g., Just \& Carpenter, 1985), and participants often report their image "jumps" in gross and piecemeal movements. In contrast, the peg task requires a relatively smooth and precise rotation so participants will not miss the point at which the pegs come into alignment. Given the results of Experiment 1, we thought a task that required a smooth rotation might play into the strengths of action-supported imagery.

Our goal for this study was to gather initial evidence that a mental model can mediate the effect of action so that action and imagery can have different spatial trajectories. There were two conditions. In the mediated-action condition, participants pulled a string attached to a spool that supported the pegboard. As they pulled the string, the pegboard rotated clockwise. In the no-action condition, participants imagined rotating the pegboard clockwise without pulling the string. Our prediction was that the mediatedaction condition would yield better performances. Although the pulling motion was a translation and the pegboard's movement was a rotation, we thought participants' mental model of the spool could help link the two motions. Consequently, the action could improve imagery for the pegboard, just as it had done for the blocks in Experiment 1. We did not have a priori predictions whether the facilitation would appear in latency or accuracy.

\section{Method}

Participants. Eight women and 8 men volunteered to participate.

Materials. The pegboard was a wooden isosceles triangle with a base of $12.6 \mathrm{~cm}$ opposite a vertex of $64^{\circ}$. Drilled into the wood, parallel to the base, were rows of holes as shown in Figure 3. The triangular shape and the number of holes were not theoretically motivated. The apparatus was a familiar peg game found in a local restaurant. Three pegs were set into one of two configurations. Figure 3 shows one configuration. For this configuration, participants had to mentally rotate the pegboard $150^{\circ}$ to align the specified pegs. The second configuration required a $100^{\circ}$ rotation and started with the point of the triangle (instead of the base) facing the participant. For both configurations, we made two versions by changing the peg colors. The color shifts masked spatially identical problems administered in the mediated-action and no-action conditions.

The pegboard rested on a flat spool $10.5 \mathrm{~cm}$ in diameter held in place by a spindle (see Figure 1). The spool had a string wrapped around its circumference. Participants sat with the spool at desk height so the center of the triangle was directly before them. By pulling the string to the right across the desk, the spool and pegboard rotated clockwise. For all the problems, regardless of condition, participants mentally rotated the pegboard clockwise.

Design. The study used a $2 \times 2$ within-subject design with three dependent measures. One measure was how long it took participants to complete a rotation. The second measure recorded how long it took participants to subsequently judge the relative direction of two pegs once they were named. The third measure was the degree of error in participants' judgments. We did not formally measure how far participants physically turned the spool with the string, because our primary interest is in the contrast between conditions and not participants' ability to turn a spool to a specific angle without much training. (Anecdotally, pull accuracy appeared normally distributed and unrelated to participants' judgments.)

The Modality factor determined whether participants pulled the string or simply imagined the pegs turning. The Configuration factor asked participants to complete the task twice within each modality (the $100^{\circ}$ and $150^{\circ}$ configurations). We kept the number of trials small because we were afraid that participants would discover a "propositional cheat" if given time. The order of trials was random with the constraint that same configuration problems did not follow one another.

Procedure. The experimenter introduced the apparatus with pegs placed at the comers of the triangle. The experimenter explained that the first part of the task was to rotate the board so that a specific peg (e.g., green) would be in the participants' line of sight to another specific peg (e.g., red). The participants pulled the string to align the pegs. Next, the experimenter named two pegs and showed how to indicate their relative direction with a ruler. The participants practiced by placing the ruler on the table wherever they wanted, as long as it was parallel with the named pegs. After completing the demonstration with eyes open, the participants practiced the task without vision. They first encoded the configuration and colors of the pegs. When ready, they grabbed the string and closed their eyes. The experimenter provided the alignment cue (e.g., "put the green before the red"). Participants pulled the string directly to their right until they thought the pegs were in alignment. They stated when they were through, and they immediately received the names of two pegs. They judged the direction of the two pegs by placing the ruler. Afterward, they opened their eyes to see how well they had done. The experimenter explained that there was a premium on speed and on the accuracy of the ruler placement relative to where the pegs should be (not how far the participant actually pulled them). The experimenter rewound the spool and placed the pegboard in another orientation. Participants completed the same sequence but did not pull the string. After completing the two practice runs, the participants completed the four timed trials. Timed trials were identical to the practice trials except the participants learned whether they should pull after they closed their eyes. When told it was a trial without 
pulling, some participants dropped the string, others held on. The measure of rotation time started with the last word of the instructions and ended when the participants indicated they were through rotating. The judgment time began with the experimenter's first word naming the target pegs and finished when the ruler was set on the table.

\section{Results}

Pulling the string improved participants' performance on the pegboard task. Figure 4 shows that participants completed the rotation and judgment of relative direction faster in the mediatedaction condition. Figure 5 shows that participants were also more accurate in the mediated-action condition. The absolute errors indicate the average size of error. The signed errors indicate the average directional size of error. A positive error means that participants placed the ruler as though they had rotated the pegs too far. For the mediated-action condition, errors were normally distributed about the correct judgment and canceled one another. The no-action condition showed a positive bias that probably resulted from configural effects; participants became confused about the relative side of one of the pegs during rotation or judgment.

Modality and Configuration constituted crossed factors in a quadruply repeated measure analysis. The four measures were rotation latency, judgment latency, degree of absolute error, and signed error. The effect of Modality showed multivariate significance, $F(4,12)=7.3$, Hotelling's $T=2.4$. Modality had a significant effect on each measurement taken separately: rotation, $F(1,15)=6.7, M S E=4.6$; judgment, $F(1,15)=13.7$, $M S E=4.4$; absolute error, $F(1,15)=9.4, M S E=1,885.6$; and signed error, $F(1,15)=7.1, M S E=2,252.3$. The Configuration factor did not generate a significant main effect or interact with Modality (multivariate $F \mathrm{~s}<1.2$ ). The lack of a Configuration effect does not imply that participants did not use mental rotations. The two levels of the Configuration factor had differences besides the angle of rotation, including the orientation of the triangle and

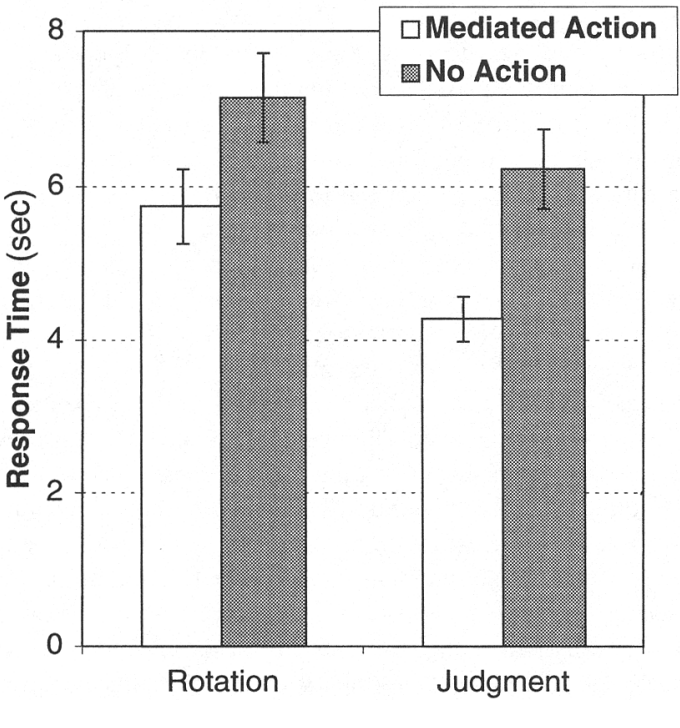

Phase of Task

Figure 4. Latencies for the rotation and judgment phases of Experiment 2 . $\mathrm{sec}=$ seconds.

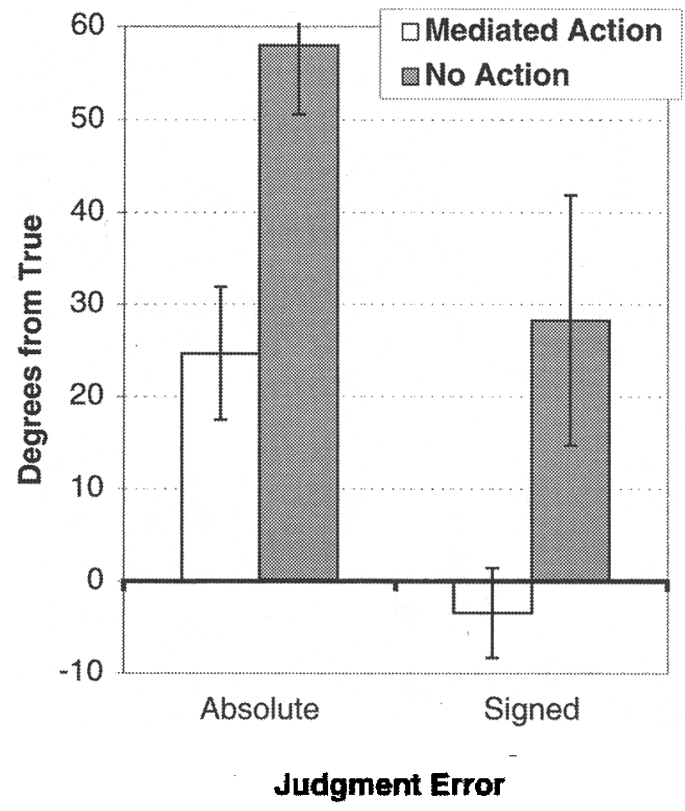

Figure 5. Errors in judgments of the relative direction of the pegs in Experiment 2.

the configuration of the pegs. So, even though the mediated-action condition rotations were $1.2 \mathrm{~s}(S D=3.2)$ faster for the $100^{\circ}$ problems than the $150^{\circ}$ problems, and the no-action condition showed little difference $(M=0.3, S D=3.2)$, it is unwise to interpret these results theoretically.

For a second view of the data, we examined whether a trial exhibited better performance in the mediated-action condition than its counterpart in the no-action condition. With 16 participants completing two trials in each modality, there were 32 pairwise comparisons. For the rotations, $63 \%$ of the trials were faster in the mediated-action condition. For the judgments (and total task), $75 \%$ of the trials were faster in the mediated-action condition. With respect to accuracy, $72 \%$ of the trials were more accurate in the mediated-action condition. It appears that the no-action condition caused participants to trade between accuracy and speed. Only 1 participant was both faster and more accurate on a no-action trial than a corresponding mediated-action trial. It also seems probable that participants traded rotation and judgment speed in the noaction condition such that they completed some of the imagery transformations during the judgment phase instead of during the rotation phase.

\section{Discussion}

Pulling a string in a translating movement facilitated participants' ability to imagine the rotation of a board of pegs. Participants were faster and more accurate when they pulled than when they tried the task without physical movement. As in Experiment 1 , this result indicates that action can interact with imagery. It also indicates that the connection between action and representation cannot be ascribed to a spatial isomorphism between participants' motions and their imagery transformations. Participants did not turn their hands in a circle to mimic the intended circular movement of the pegboard. 
The pegboard task took substantially more time than the block rotation task of Experiment 1, with total latencies averaging around $10 \mathrm{~s}$ instead of $7 \mathrm{~s}$. Some of this difference may be due to the limited practice and the added component of placing a ruler on the table to indicate the angle. As such, it is difficult to compare the relative effects of action across experiments. Regardless, one may note that in the pegboard task, action decreased total latencies by about $3 \mathrm{~s}$, whereas actually turning the block in Experiment 1 reduced latencies by about $1 \mathrm{~s}$. The basis of the difference is open to future research.

The pegboard results suggest that participants' model of the spool system made it possible for them to convert their linear physical action into the mental rotation of the pegboard. Before we consider the characteristics of the mental models that enable this conversion, it is important to eliminate an alternative explanation of the results. This explanation is that participants did not depend on a model to reap the benefits of action. Instead, the general action of moving their hand in a noninterfering manner may have facilitated performance. Perhaps the gestures primed the retrieval of the different orientations of the board (e.g., Rauscher, Krauss, \& Chen, 1996). Experiment 3 tried to show the significance of participants' mental models directly. In this experiment, participants always took the same action but with different models in mind.

\section{Experiment 3}

Experiments 1 and 2 used facilitation paradigms to examine the effects of action on imagery. The present study used an interference paradigm. Our goal was to show that action enhances or disrupts a desired imagery transformation depending on a participant's mental model. The experiment used the block rotation task of Experiment 1 but placed the block on the spool from Experiment 2. By flipping the spool over, the exact same movement of pulling the string to the right could cause the spool to turn clockwise or counterclockwise. Figure 6 outlines the experimental setup and design.

For each trial, participants imagined the block turning clockwise or counterclockwise $90^{\circ}$. To encourage the appropriate mental

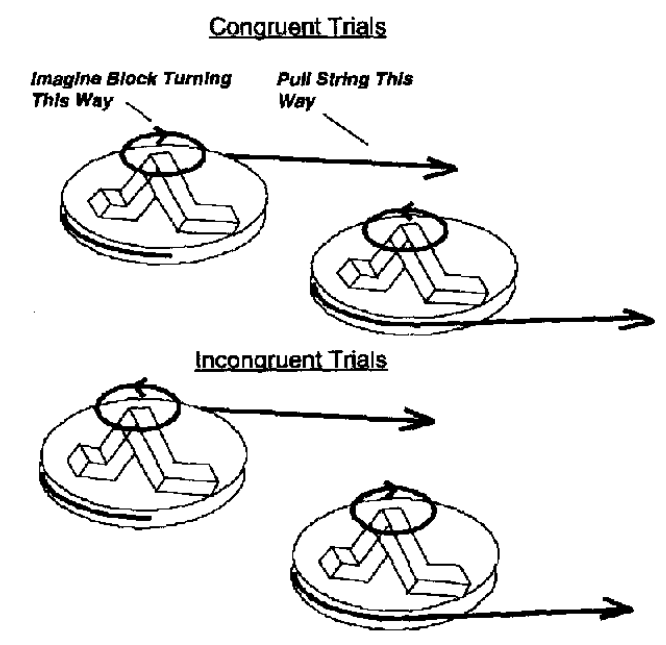

Figure 6. The pulling tasks of Experiment 3. rotation, the experimenter told them that for most of the trials, the target block would appear in the expected position (e.g., rotated clockwise $90^{\circ}$ ). For a congruent trial, participants saw that pulling the string would turn the spool in one direction (e.g., clockwise), and they were told an expected direction that corresponded with the spool's movement (e.g., clockwise $90^{\circ}$ ). For an incongruent trial, they were told an expected direction that was opposite of the spool's movement (e.g., counterclockwise 90\%). In either case, the participants had to pull the string once they received directions. If a mental model mediates the effect of action on imagery, then the incongruent trials should be more difficult than the congruent trials. Participants "know" they are pulling the block in one direction and this drives their imagery, yet they have to imagine the block turning the other direction. Alternatively, if action effects do not depend on a model, then there should be limited differences between the congruent and incongruent trials.

During the rotations, participants closed their eyes so they could not see the block and spool. Yet, it was possible that participants might feel the clockwise or counterclockwise motion of the spool through the string. If this were the case, then difficulties in implementing the incongruent trials could be the result of perceptual interference; participants try to imagine the block turning one direction but they feel it turning the other. We want to claim, however, that participants generate the interference through their models. To support this claim, we have to show that participants do not perceive the direction of the spool when pulling. A second group of participants saw the spool apparatus. They grabbed the string and closed their eyes. The experimenter lifted the spool from its spindle and placed it back down. Participants pulled the string to determine whether the spool turned clockwise or counterclockwise. If pulling does not provide information about the turning direction, participants should perform at chance.

\section{Method}

Participants. Twelve adults ( 8 women and 4 men) volunteered to participate in the main study. Another 3 men and 3 women were in the perception study.

Design. The primary experiment included two within-subject factors. One factor was whether the pull direction and imagine direction were congruent or incongruent. Half of the pulls were clockwise and half were counterclockwise. Crossed with this, half of the imagined rotations were clockwise and half were counterclockwise. For a congruent trial, participants pulled the string so the block rotated in the same direction as they were told to imagine it rotating $90^{\circ}$ (pull-clock/imagine-clock or pullcounter/imagine-counter). For an incongruent trial, participants pulled the string so the block turned one direction while they tried to imagine it turning the other direction by (pull-clock/imagine-counter or pull-counter/ imagine-clock). The second factor was whether the target block appeared at the expected or original position. As before, rotation and match times were both recorded, and incorrect trials plus lost-image trials were replaced at the end of the session. Reflected trials occurred randomly on one fourth of the trials to prevent participants from saying "same" without completing the task. Because we expected the interference paradigm to introduce extra variability, we doubled the number of trials by using two sets of block shapes. All told, participants completed 16 correct, same trials (2 pull directions $\times 2$ imagined directions $\times 2$ target positions $\times 2$ blocks).

For the perception study, participants completed 20 pulls. Ten pulls turned the spool clockwise, and 10 pulls turned the spool counterclockwise. The clock and counter trials were randomly mixed. Participants tried to determine the direction the spool turned without looking. 
Procedure. The main experiment included the usual four phases. During the encoding phase, participants studied a block on the spool. They also pulled the end of the string just enough to make sure they noticed which way the spool turned. When ready, they closed their eyes. The experimenter lowered the shelf and placed the target block in position. During the subsequent precue phase, the experimenter told participants to imagine the block turned to either $90^{\circ}$ clockwise or counterclockwise. The completion of this directive started the rotation phase. Upon hearing the direction of imagined rotation, participants started to pull the string. At the same time, they were supposed to imagine the block rotating to the expected position. Once they had mentally rotated the target block to its position, they opened their eyes and completed the match phase. Afterward, participants looked at the blocks for feedback.

To train participants for the full sequence, we first made them practice encoding the block. They then practiced pulling the string until the block had turned $90^{\circ}$ (practicing both directions equally). They then practiced turning the block $90^{\circ}$ with their eyes closed. Afterward, they completed the rotation phase and tried to match their image of the block against the target on the shelf. Finally, they were told that they were to imagine the block according to the verbal directions, but they were to pull the string at the same time (whether or not it matched the verbal directions). They did a few congruent trials and a few incongruent trials before the actual timed trials. Participants began the timed trials once they were fluent with the complete sequence.

In the perception study, participants first pulled the string a few times with their eyes open for both the clockwise and counterclockwise spool positions. For the recorded trials, they held the string and closed their eyes. The experimenter lifted the spool from the spindle and then replaced it in the clock or counter position. Participants pulled the string a few inches (approximately a $90^{\circ}$ turn of the spool). They tried to determine the spool's direction of movement. They opened their eyes to see the correct answer.

\section{Results}

Perception study. Simply pulling the string did not render the direction of spool rotation. Participants' average performance was close to chance $(M=53 \%, S D=14 \%)$. At the two ends of the distribution, 1 participant was $70 \%$ correct and 1 participant was $35 \%$ correct. Perhaps the most accurate participant was more sensitive to the available information. Regardless, pulling the string did not generally provide sufficient information for participants to feel the direction of the spool.

Imagination study. Errors occurred on approximately $10 \%$ of the trials. Nine of the 12 participants made errors. The errors were distributed across the expected and original trials (12\% and $8 \%$, respectively). The errors were also distributed across the congruent and incongruent trials ( $9 \%$ and $11 \%$, respectively).

Figure 7 displays the rotation and match times broken out by congruency and target condition. Participants completed the rotations faster in the congruent condition than in the incongruent condition. This supports the claim that a model of a tool can mediate the effect of action on imagery. Participants completed the match faster when the target was in the expected position than in the original position. This indicates that participants were indeed transforming their mental representation before opening their eyes. A within-subject analysis of variance compared rotation and match times for the crossed factors of Congruency and Target. There was a significant effect of Congruency on rotation time, $F(1$, $11)=57.0, M S E=2.4$, but not on match time, $F(1,11)=0.2$, $M S E=1.8$. There was a significant effect of Target on match time, $F(1,11)=9.4, M S E=2.7$, but not on rotation time, $F(1$, $11)=0.0, M S E=0.8$. There were no interaction effects, $F(2$,

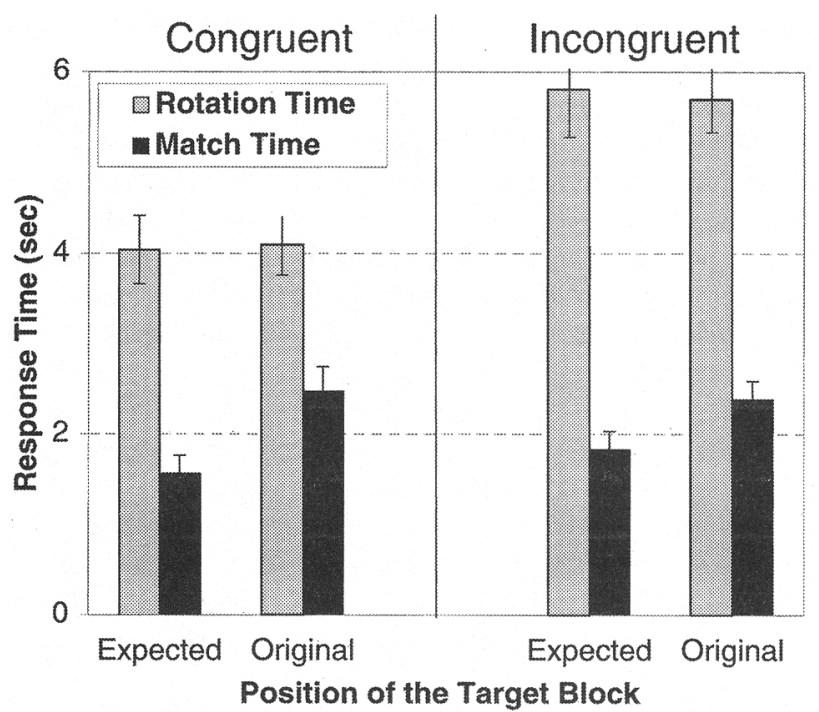

Figure 7. Rotation and match latencies for Experiment 3.

$10)=1.1$. There were no effects for the direction of spool rotation, imagined rotation, or the two block shapes (all $F \mathrm{~s}<2.0$ ).

Of the 96 total pairs of trials, $69 \%$ followed the predicted pattern in which an expected trial was faster than the equivalent original trial. To compare congruent and incongruent trials, we paired trials that had the same pull direction but different imagine directions (e.g., pull-clock/imagine-clock vs. pull-clock/imagine-counter). Of the 96 pairs, $84 \%$ fit the predicted pattern in which a congruent rotation was faster than its yoked incongruent rotation.

In addition to rotation times, participants' comments indicated they had difficulty with the incongruent condition. Upon introduction to the incongruent task during training, most participants spontaneously commented that it was difficult. Upon debriefing, participants described their attempts to overcome the interference. Most mentioned several methods, but none seemed satisfied with any of their solutions. Some tried to avoid the interference altogether; they first imagined the block turning with the spool, and then after they had pulled the string an appropriate distance, they imagined rotating the block $180^{\circ}$ back to the expected position. Many tried to refashion their mental model of the situation. Some attempted to imagine the spool rotating one way with the block rotating in a detached fashion in the other. Some tried to imagine a hidden mechanism that made the spool turn the opposite direction on incongruent trials. Similarly, some stated that they tried to reattach the string to the other side of the spool in their imagination but that it was difficult to do, especially because they started pulling the string immediately upon the imagination direction. This difficulty fits other research that found that people have difficulty changing the structure of an analog mental model once it is in the process of a continuous transformation (Schwartz, 1999). Finally, most participants said that at one time they tried to ignore their pulling in the incongruent condition but had a difficult time doing so.

\section{Discussion}

The results provide two bits of information. The first is that the exact same action can have opposing effects on the exact same 
imagined target motion. When participants maintained a model in which their pulling turned the block one way and they had to imagine the block going the other, they had trouble completing the task. Yet, when their model fit the direction of imagined rotation they did not have trouble, although the action was identical.

The second bit of information is that participants can have trouble disregarding their actions (see Farrell \& Robertson, 1998). In the incongruent condition, participants stated they could not ignore their motor activity. A cross-experimental comparison supports this introspection. If participants ignored their actions, then they would simply imagine the block rotating to the target orientation. In this case, the rotation latencies should be comparable with those of the no-action condition in Experiment 1 . In that study, participants only imagined the rotation of the block. The latencies for the incongruent condition were roughly $1.5 \mathrm{~s}$ longer than latencies for the no-action condition.

It is interesting that participants could not abandon their models when they interfered with the task of rotating a block. Although some said they tried, few participants uncoupled the effect of action on their representation or reconstructed their model so that the action would facilitate the target rotation. One possible explanation is that the perception of the spool at the start of each trial provided an imagery trace that was too great to overcome once the eyes were closed. Yet, it seems likely that the same results could occur despite visual perception. For example, participants might encode the block and spool. Once they close their eyes, the experimenter could make an appropriate noise and say that the spool had been flipped over. The resulting model, although opposite in implication to the visual perception, may have effects of equal strength. This prediction gains credibility in light of Rieser et al.'s (1994) study, which found that perception is not necessary to entrench a mental model. Children, while at home, could successfully imagine themselves at different locations in their classrooms, as measured by their ability to make judgments of relative direction (e.g., where is the teacher's desk from where you are facing?). Yet, once they adopted the perspective of a specific location in the classroom (e.g., facing the front door), they had difficulty changing to a new perspective within the room (e.g., facing the chalkboard). This result directly indicates that perception is not necessary to fixate a mental model. The causes and parameters of fixation, however, remain an open question.

\section{General Discussion}

There is evidence that action can influence perception. Coren (1986), for example, showed that saccadic eye movements are responsible for the Mueller-Lyer optical illusion. The present experiments offered evidence that action can also influence the imagination. All three experiments demonstrated that hand movements affect participants' ability to imagine objects turned to different orientations. Yet, despite the frequent comparison with perception (Finke, 1980; Pylyshyn, 1981; Shepard, 1984), imagery representations are not the same thing as perceptions. Imagery representations are less constrained by environmental input and more open to the "imagination." Consequently, they permit models of the tools at hand to mediate the effects of action on spatial updating. Experiments 2 and 3 demonstrated that spatial actions do not have to yield spatially isomorphic effects on the imagination. Pulling a string in a translation movement helped participants imagine the rotation of an object. Experiment 3 demonstrated that participants' model of the device was responsible for this effect. The exact same pulling motion facilitated or interfered with the exact same target rotation depending on whether people thought their pull would turn the spool clockwise or counterclockwise. Similar to demonstrations with language comprehension (Bransford \& Johnson, 1972), it is not simply properties of the stimulus or people's parsing mechanisms that determine the trajectory of cognition but also the model people have in mind. The coupling of action and representation is subject to dynamic reformulation depending on people's knowledge and beliefs.

The present results indicate the importance of learning for connecting action and thought (see also Iriki, Tanaka, \& Iwamura, 1996; Rieser, Pick, Ashmead, \& Garing, 1995). By this, we do not mean to imply that bodily action necessarily serves as a basis for learning higher order thought, as proposed by theorists including Piaget (1954) and Thelen and Smith (1994). The present experiments did not explore the effects of action on learning or reasoning, although it seems possible that the models that support tool use play a role in deduction (Johnson-Laird, 1983). Instead, the experiments highlighted that learned knowledge, rather than an invariant geometric mapping, mediates the effect of action on imagery. In the following, we review possible constraints that may circumscribe the effects of learning, and then we consider the topic of transfer to highlight the uniqueness of tool models in the cognitive pantheon.

We examine four possible constraints that may limit the couplings of action and imagery. One possibility is that people may not be able to couple an action with an imagined update in the opposing direction. For example, it may be impossible for a clockwise hand motion to facilitate imagery for a counterclockwise object rotation (e.g., Wohlschlåger \& Wolschlåger, 1998). Alternatively, according to the hypothesis of timing-responsive representations, it should be possible to couple opposed movements because it is the timing of an action rather than its direction that provides the impetus for representational change. ${ }^{1}$ Given sufficient experience with an appropriate tool, people should be able to surmount problems with spatial incompatibility (see Prinz, 1997). For example, if people master the behavior of two meshing gears, then a clockwise action over one gear may help them imagine the counterclockwise motion of the second gear.

A second constraint might be that mental models only mediate couplings of action and imagery in the context of manipulation and not in the context of navigation. For navigation, people may rely on a direct spatial mapping to coordinate their physical footsteps with their updates of position. Good evidence indicates that multiple species, for example, ants, exploit a mapping between the spatial properties of their movements and their estimates of loca-

\footnotetext{
${ }^{1}$ Although we propose that the direction of movement is not the facilitating component of action, we do not know whether timing without action, and therefore without spatial movement, would facilitate imagery transformations. For example, would passively listening to a metronome facilitate imagery transformations? The present results suggest that before there will be an effect, if there is one, people need a mental model that associates the metronome's timing with the target spatial updates. Without a model of association, listening to a metronome would be no better than simply moving one's arm to the right while looking at a block sitting on a table (without any thoughts of a spool or string).
} 
tion (Wehner \& Srinivasan, 1981). Yet, people can also use tools, such as bicycles and skis, to aid their locomotion. People may learn to model the tools that facilitate movement in large-scale space and use these models to mediate the effects of action on the imagination. Studies with blindfolded cyclists might reveal that their mental updates of position depend on the force required to turn the pedals or the gear they believe they are using.

The final two constraints we consider are two types of tool complexity. One complexity involves the outcome of the tool. It is clear that some tools produce object behaviors too complex to imagine. But, assuming a manageable imagery task, is there a complexity of outcome that action cannot facilitate? We anticipate that future research will indicate an effect of action on nonspatial imagery (e.g., with sound and musical instruments), and therefore, a general characterization of complexity should not be limited to spatial terms. We suggest psychological complexity is a function of the number of changes in tool output associated with a single moment of action. We doubt there is a strong effect of action for a tool that uses a button press to initiate a complex sequence of changing movements. However, for a tool that yields a one-to-one correspondence between moments of action and moments of outcome, then people should exhibit facilitating effects of action. What counts as a moment of change for different modalities is an empirical question for future research.

The second complexity is the mechanism of the tool. For the spool, it is possible that participants modeled the actual mechanical linkage by envisioning the string moving about the spool. More complicated tools, however, may make it cumbersome or impossible to visualize the mechanisms that mediate between action and outcome. Although the inability to visualize the internal mechanisms may make it difficult for people to understand the tool, it should not prevent their ability to link action and imagination with a model of the tool. Adults, for example, can model the effects of steering a car, but they do not know the mechanisms that convert a steering motion into a car turn. In the present task, if the string threaded a circuitous route from the spool to one's hand, we predict that people would not have to imagine all the bends and turns of the string to reap the benefits of pulling. The limit on the model people can use to couple action and imagery probably has more to do with their experience of the functional input-output characteristics of a tool than with their ability to visualize or understand the mechanism that achieves those functions. The significance of mental models for the current story is not their potential for mechanical understanding. Once invoked, it is conceivable that an overlearned model becomes little more than an implicit transformation matrix that converts action into imagery updates, inaccessible to conceptual or visual analysis (Schwartz \& Black, 1999). What is significant here is the ability of models to dynamically reformulate the coupling between action and imagination depending on which model people bring to mind.

The ability to insert models between one's action and imagined spatial update presents a relatively unique form of cognition. One way to appreciate the special nature of tool models is to consider a potential experiment on transfer. When individuals mentally rotate blocks for numerous trials, their latencies decrease (Kail, 1986; Kaushall \& Parsons, 1981; Tarr \& Pinker, 1989). To our knowledge, there is little evidence that people transfer these performance improvements with blocks to a new rotation problem like the pegboard task. Imagine, however, that a group of people spend numerous trials learning the behavior of a novel tool. For example, they might push a lever that rotates a turntable. Over time, they will develop a robust model of the lever-turntable tool. What will happen when these people complete the pegboard rotation task? If they imagine rotating the pegboard in the context of pushing the lever, their action should facilitate their mental rotations. This is what happened with the spool in Experiment 2. Although the pegboard task was novel, participants transferred their knowledge of the spool to facilitate the imagined rotation of the pegs.

Pulling the string in Experiment 2 reduced latencies by roughly $3 \mathrm{~s}$ and cut errors in half compared with the no-action condition. We assume the lever tool would do the same given sufficient experience. We also assume that this facilitation would be greater than if participants had spent thousands of trials practicing block rotations before meeting the pegboard. If this thought experiment turns out to be correct, it yields an unusual transfer result. There is more transfer across two dissimilar situations (learning a lever device and rotating a pegboard) compared with two similar situations (learning to rotate a block and rotating a pegboard). If true, this suggests the special status of tool imagery, because it does not follow the same rules as other psychological functions that primarily depend on similarity for their operation.

Operations including analogy, identification, and retrieval depend on the similarity between the source and the target for prior knowledge to have an effect (see Vosniadou \& Ortony, 1989). The introduction highlighted that research on action and representation presumes that similarity also explains the effect of action on imagery. People reap the benefits of well-learned spatial actions or motor plans because their mental transformation takes a similar trajectory. With tools, however, people do not have to rely on direct similarities. They can apply well-learned models of tool operations to help them convert one motion (their action) into another (an imagined environmental transformation). So, rather than making thought subordinate to preexisting spatial similarities, people use models to help them overcome a lack of similarity. They construct a mental "hinge" that connects otherwise dissimilar motions. Just as tools help people change the physical environment to suit their needs, models of tools help people change the effects of action on their mental environment. We propose that this similarity is more than coincidence.

\section{References}

Bransford, J. D., \& Johnson, M. K. (1972). Contextual prerequisites for understanding: Some investigations of comprehension and recall. Journal of Verbal Learning and Verbal Behavior, 11, 717-726.

Cooper, L. A., \& Podgorny, P. (1976). Mental transformations and visual comparison process: Effects of complexity and similarity. Journal of Experimental Psychology: Human Perception and Performance, 2, 503514.

Coren, S. (1986). An efferent component in the visual perception of direction and extent. Psychological Review, 93, 391-410.

Decety, J., \& Michel, F. (1989). Comparative analysis of actual and mental movement times in two graphic tasks. Brain and Cognition. 11, 87-97. de'Sperati, C. \& Stucchi, N. (1997), Recognizing the motion of a graspable object is guided by handedness. Cognitive Neuroscience and Neuropsychology, 8, 2761-2765.

Diamond, J. M. (1997). Guns, germs, and steel: The fates of human societies. New York: Norton. 
Farrell, M. J., \& Robertson, I. H. (1998). Mental rotation and the automatic updating of body-centered spatial relationships. Journal of Experimental Psychology: Learning, Memory, and Cognition, 24, 227-233.

Finke, R. A. (1980). Levels of equivalence in imagery and perception. Psychological Review, 87, 113-132.

Gallistel, C. R. (1990). The organization of learning. Cambridge, MA: MIT Press.

Hegarty, M. (1992). Mental animation: Inferring motion from static displays of mechanical systems. Journal of Experimental Psychology: Learning, Memory, and Cognition, 18, 1084-1102.

Iriki, A., Tanaka, M., \& Iwamura, Y. (1996). Coding of modified body schema during tool use by macaque postcentral neurones. Neuroreport 7, 2325-2330.

Jeannerod, M. (1994). The representing brain: Neural correlates of motor intention and imagery. Behavioral and Brain Sciences, 17, 187-245.

Johnson-Laird, P. N. (1983). Mental models: Towards a cognitive science of language, inference and consciousness. Cambridge, England: Cambridge University Press.

Just, M. A., \& Carpenter, P. A. (1985). Cognitive coordinate systems: Accounts of mental rotation and individual differences in spatial ability. Psychological Review, 92, 137-172.

Kail, R. (1986). The impact of extended practice on the rate of mental rotation. Journal of Experimental Child Psychology, 42, 378-391.

Kaushall, P., \& Parsons, L. M. (1981). Optical information and practice in the discrimination of 3-D mirror-reflected objects. Perception, 10, 545562.

Kohler, I. (1964). The formation and transformation of the perceptual world (Harry Fiss, Trans.). Psychological Issues, 3(4, Monograph No. 12).

Krist, H., Fieberg, E. L., \& Wilkening, F. (1993). Intuitive physics in action and judgment: The development of knowledge about projectile motion. Journal of Experimental Psychology: Learning, Memory, and Cognition, 19, 952-966.

Parsons, L. M. (1994). Temporal and kinematic properties of motor behavior reflected in mentally simulated action. Journal of Experimental Psychology: Human Perception and Performance, 20, 709-730.

Piaget, J. (1954). The construction of reality in the child. New York: Basic Books.

Prinz, W. (1997). Why Donders has led us astray. In B. Hommel \& W. Prinz (Eds). Theoretical issues in stimulus-response compatibility (247268). New York: Elsevier.

Pylyshyn, Z. W. (1981). The imagery debate: Analog media versus tacit knowledge. In N. Block (Ed.), Imagery (pp. 151-206). Cambridge, MA: MIT Press.

Rauscher, F. H., Krauss, R. M., \& Chen, Y. (1996). Gesture, speech, and lexical access: The role of lexical movements in speech production. Psychological Science, 7, 226-231.

Rieser, J. J., Garing, A. E., \& Young, M. E. (1994). Imagery, action, and young children's spatial orientation. It's not being there that counts, it's what one has in mind. Child Development, 65, 1262-1278.

Rieser, J. J., Pick, H. L., Jr., Ashmead, D. H., \& Garing, A. E. (1995). The calibration of human locomotion and models of perceptual-motor organization. Journal of Experimental Psychology: Human Perception and Performance, 21, 480-497.
Schwartz, D. L. (1999). Physical imagery: Kinematic versus dynamic models. Cognitive Psychology, 38, 433-464.

Schwartz, D. L., \& Berry, D. (2000). Real-time cognition: Coordinating physical and symbolic activity. Manuscript submitted for publication.

Schwartz, D. L., \& Black, J. B. (1996). Analog imagery in mental model reasoning: Depictive models. Cognitive Psychology, 30, 154-219.

Schwartz, D. L., \& Black, T. (1999). Inferences through imagined actions: knowing by simulated doing. Journal of Experimental Psychology: Learning, Memory, and Cognition., 25, 116-136.

Sheerer, E. (1984). Motor theories of cognitive structure: A historical review. In W. Prinz \& A. F. Sanders (Eds.), Cognition and motor processes (pp. 77-97). Berlin: Springer.

Shelton, A. L., \& McNamara, T. P. (1997). Muitiple views of spatial memory. Psychonomic Bulletin \& Review, 4, 102-106.

Shepard, R. N. (1984). Ecological constraints on internal representation: Resonant kinematics of perceiving, imagining, thinking, and dreaming. Psychological Review, 91, 417-447.

Shepard, R. N., \& Cooper, L. A. (Eds.). (1986). Mental images and their transformation. Cambridge, MA: MIT Press.

Shepard, R. N., \& Metzler, J. (1971). Mental rotation of three dimensional objects. Science, 171, 701-703.

Simons, D. J., \& Wang, R. F. (1998). Perceiving real-world viewpoint changes. Psychological Science, 9, 315-320.

Tarr, M. J., \& Pinker, S. (1989). Mental rotation and orientationdependence in shape recognition. Cognitive Psychology, 21, 233-282.

Thelen, E., \& Smith, L. B. (1994). A dynamic systems approach to the development of cognition and action. Cambridge, MA: MIT Press.

Turvey, M. T., Solomon, H. Y., \& Burton, G. (1989). An ecological analysis of knowing by wielding. Journal of the Experimental Analysis of Behovior, 52, 387-407.

Vosniadou, S., \& Ortony, A. (Eds.). (1989). Similarity and analogical reasoning. New York: Cambridge University Press.

Wang, R. F., \& Simons, D. J. (1999). Active and passive scene recognition across views. Cognition, 70, 191-210.

Wehner, R., \& Srinivasan, M. V. (1981). Searching behavior in desert ants, genus Cataglyphis (Formicidae, Hymenoptera). Journal of Comparative Physiology, 142, 315-338.

Wexler, M., \& Klam, F. (in press). Movement prediction and movement production. Joumal of Experimental Psychology: Human Perception and Performance.

Wexler, M., Kosslyn, S. M., \& Berthoz, A. (1998). Motor processes in mental rotation. Cognition, 68, 77-94.

Wohlschlåger, A., \& Wolschlåger, A. (1998). Mental and manual rotation. Journal of Experimental Psychology: Human Perception and Performance, 24, 397-412.

Wraga, M., Creem, S. H., \& Proffitt, D. R. (2000). Updating displays after imagined object and viewer rotations. Journal of Experimental Psychology: Learning, Memory, and Cognition, 26, 151-168.

Received December 20, 1999

Revision received July 10,2000

Accepted July 10, 2000 